\title{
An Information Systems Profession to Meet the Challenge of the 2000s
}

\author{
Ronald Stamper ${ }^{1}$
}

Received January 5, 1994; accepted February 19, 1995

\begin{abstract}
Technical proficiency has not enabled our profession to deliver value for money in terms of improved organisational performance. Evidence of this failure is adduced. The recommended change is to approach organisations as the information systems we develop. To achieve this we need a better understanding of information, the key resource, better ways of modelling organisations in information terms, and new tools for analysis, design, and systems development. Semiotics, the theory of sign, provides a suitable framework in which to make "information" and related concepts more precise. A semiotics of organisations leads to modelling them as systems of social norms from which information requirements can be logically deduced. This approach yields methods and tools for analyzing and designing the social, pragmatic, and semantic aspects of information systems that receive little attention in our current methodologies.
\end{abstract}

KEY WORDS: information; profession; semiotics; organisation; semantics.

\section{INTRODUCTION}

Having been asked to contribute an article on the future direction of the information systems profession, I make no apology for making a statement of my personal convictions on this issue. There is certainly no way of making a scientific statement on this subject. I have a long-held conviction that our profession must change, and over a long period, I have, with others, directed my efforts towards formulating concepts and devising methods and tools that will be needed as our new role develops. So I shall also say something about these lines of thought as well as discussing the forces shaping our professional future.

The motive for change is the evident failure of organisational systems based on computer technology to reward many organisations that have invested in them. Evidently there is something wrong with our concepts, our approach, and/

\footnotetext{
${ }^{1}$ University of Twente, P.O. Box 217,7500 AE Enschede, The Netherlands.
} 
or our methods. Conceptually we are still embarrassingly vague about the meanings of concepts central to our work: "information" is the most obvious example. We do not yet understand the nature of information, although it is the key economic resource of the postindustrial age. Our approach has been to build computer-based information systems instead of treating the whole organisation as an information system and the appropriate object of our professional attention. The methods and tools we now deploy are almost like blinkers that keep our eyes fixed on the computer-track and prevent us from joining the serious race running on the organisational-track. We must recognise that organisations are information systems and that we need new methods and tools to deal with them. The proposed changes to our profession will not displace our technical competence but will provide a context that will improve our professional competence to serve society.

\section{MOTIVATION}

There are still many people quite satisfied with the established, technically oriented attitudes. So, first, let us look at some evidence that should shake any complacency.

The economy can be divided into two main sectors responsible for physical production and information production. In the physical sector the productivity of labour has been increasing steadily for centuries and certainly through recent decades. Our profession has a key responsibility for productivity in the information sector, where, despite the advances in information technology (IT), productivity improvement has been disappointing. For example, the impact of IT in the U.S. financial sector, analyzed by Franke (1987) (Fig. 1) shows that the productivity of labour in the industry as a whole hardly changed despite massive investments in IT, but capital productivity did fall dramatically. This industry, typical of the information sector of the economy, was ideal for IT investment, especially as the cost-performance ratio for IT was also improving rapidly.

Why is the record so disappointing? Actually the picture is confused and complicated. Perhaps the banks and insurance firms were induced to leapfrog over each other's IT investments in order to preserve competitive advantage. This can have the effect, within an industry, of inducing a find of hysterical pursuit of a mirage. Each competitive advantage can only be short-lived, and the net effect may be that the industry as a whole fails to use IT effectively. Perhaps the financial yardstick is misleading because the financial sector has been changing its nature profoundly to produce many new services that were unthinkable without IT. The output of a whole industry must be measured in money terms, but money value of services provided may be a poor indicator of the output of an information industry. Dealing with physical commodities, we 
Financial industry USA

(semilog, $1948=100$

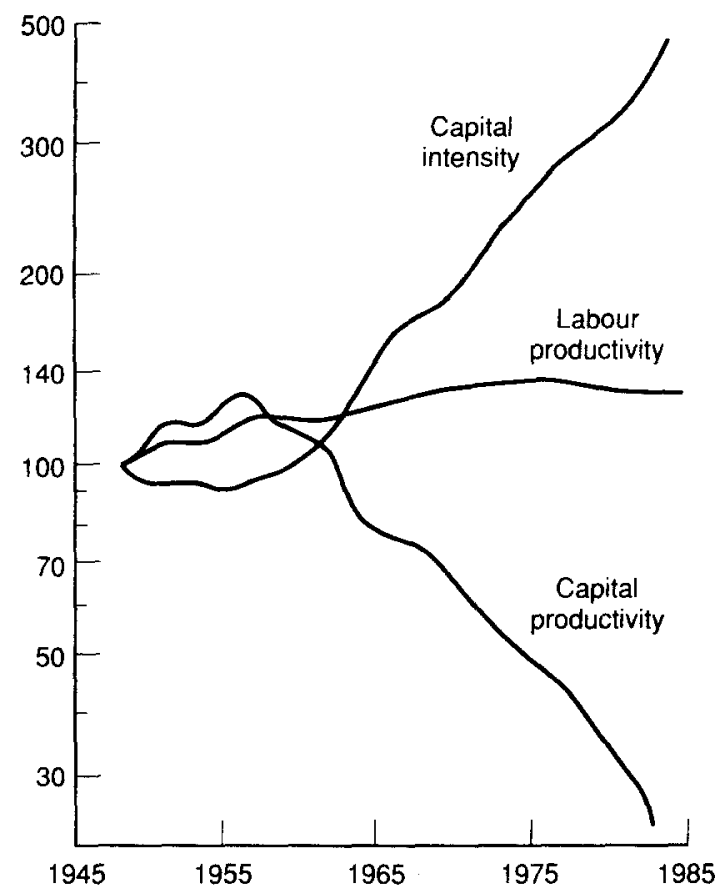

Fig. 1. The apparently disappointing productivity record for IT investments in the U.S. financial sector. Source: Franke (1987).

can always make a cross-check of the cost per ton of coal, steel, wheat, coffee, etc., but we do not understand well enough what information is to be able to make a similar nonmonetary productivity assessment. Maybe we are getting good returns on IT investments but we are unable to measure them. The disappointment may be accounted for more by our lack of understanding of the information concept than by the apparent performance of the industry.

What other evidence have we? Few people have done more than Paul Strassmann to document the generally poor return on investments in IT. His graph, reproduced in Fig. 2, shows that, for nearly 300 manufacturing companies in several countries, there is no clear relationship between company performance (return on assets) and investment in IT (as percentage of turnover). Incidentally other standard accounting yardsticks did no better.

In his 1985 book Strassmann noted similar results in a study by Cron and Sobel (1983) based on the financial results for 138 wholesale companies which, categorised as non-, medium-, and heavy users of computers, exhibited, on 


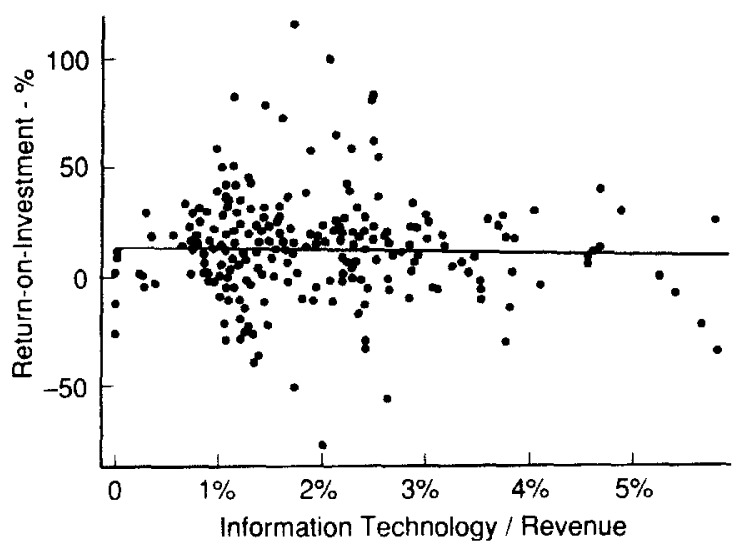

Fig. 2. Lack of correlation between business performance and IT investment. Source: Strassmann (1985).

average, a declining performance with increasing use of computers. However, dividing the sample into quartiles according to their financial performance, a more interesting story is told by these statistics; see Table I.

The unimodal distribution of performance among noncomputer users gave way to bimodal distributions for medium and heavy computer users. A plausible explanation is that well-run companies benefit from using computers, whereas badly run companies are made worse by them. Strassmann's detailed analysis supports this conjecture, as it reveals marked differences between the use of information and IT resources in the most successful and least successful companies in his sample. Our failure adequately to understand the causal relationships between computer use in different organisational circumstances and the resulting performance is a failure of professional understanding.

One causal connection between the organisation and the performance of IT investments may be observed in the effectiveness of software engineering projects. There is abundant evidence that software is often delivered late or over

Table I. Wholesalers: Performance and Computer Use (Source: Cron and Sobol, 1983)

\begin{tabular}{llcc}
\hline \multicolumn{1}{c}{ Computer use } & None & Medium & High \\
\hline Av \% return/assets & 11.3 & 9.8 & 8.8 \\
Best quartile \% & 25 & $30^{*}$ & $30^{*}$ \\
Good quartile \% & 28 & 19 & 15 \\
Poor quartile \% & $36^{*}$ & 21 & 12 \\
Worst quartile \% & 11 & $30^{*}$ & $43^{* *}$ \\
\hline
\end{tabular}


budget (Jayaratna, 1990), but that is a minor problem compared with the suitability of the software for the organisation. Many technically sound systems, when delivered, often fail to meet users' needs. Jayaratna quotes some experience in U.S. federal software projects in Fig. 3. The problem here is not one of software engineering but a lack of understanding of the organisation to be served. Because we have an inadequate knowledge of organisations as information systems, it is difficult to perceive their information needs fully and correctly and then express them suitably to the software engineer. Our methods and tools for analysis were developed in order to facilitate the introduction of computers to automate established bureaucracies rather than to solve the more fundamental problems of organisations.

To end this sampling of the evidence, let us remind ourselves of the high rate of performance increase for IT compared with other technologies. In Fig. 4, which appeared in Scott-Morton (1989), U.S. data show that IT improves about 18 times faster per year compared with a basket of six other product groups. Moreover, compared with the cost of labour, the improvement is 30\% per annum. Those trends do not seem to have altered in more recent years. With such rapidly improving technological resources at our disposal, we should be

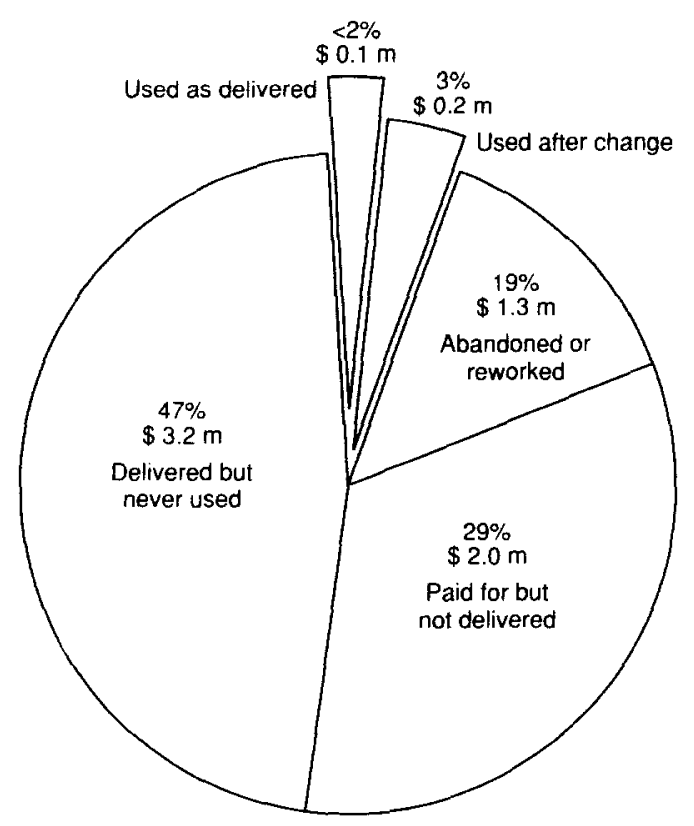

Fig. 3. (In)effectiveness of some U.S. federal software projects. The diagram represents the fate of a total software budget of $\$ 6.2$ million. 


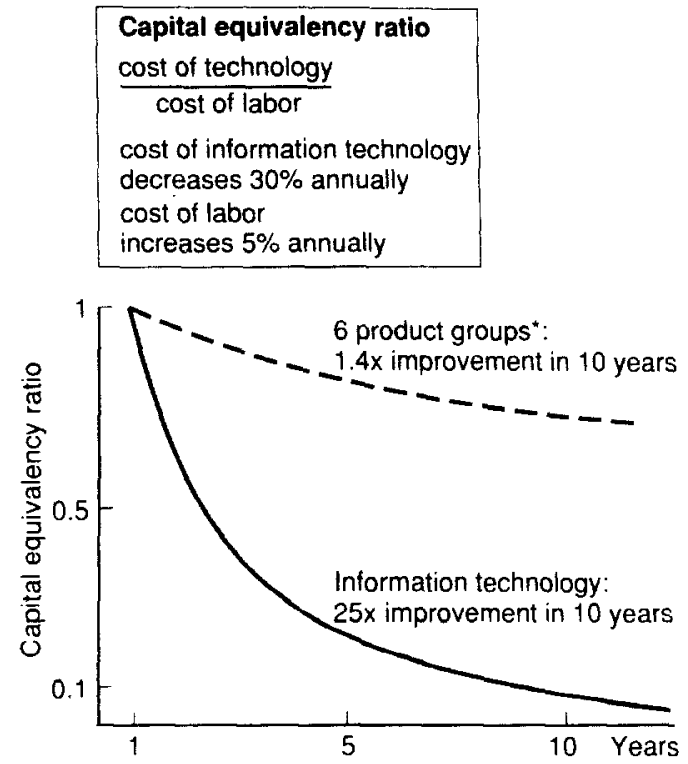

Fig. 4. Changing capital-labour ratio of IT in the United States. Source: Bureau of Labor Statistics, producer price index. Compensation per hour, nonfarm business sector, 1955-1980.

ashamed at our failure to bring dramatic improvements to our economies. The technical problems which used, quite rightly, to dominate our work are rapidly being alleviated so that the problems concerning the human and social aspects of information systems are beginning to dominate our work.

There are many more illustrations of our failure to meet our professional obligations to society. To summarise the above remarks, we should be looking at the business performance of our information systems rather than being content with the technical performance of our data systems; a combined knowledge of organisations and technology is called for, especially an improved ability to express organisational needs; we have to deliver systems on time, on budget and able to meet actual user needs; our line of vision should be raised more often to the scale of an economy rather than focusing no higher than a subsystem of an individual enterprise; we should develop a far more mature understanding of information as an economic resource; and we should be more modest about trumpeting the technical achievements of IT until we can consistently translate them into real economic values.

\section{TECHNOLOGY IN ITS SOCIAL CONTEXT}

IT has no real economic value in its own right. Only in its social, organisational, and economic context can it play a role in improving the quality and 
supply of goods and services. Until we can place the technical systems we create in an ambient social structure that can make this translation, the performance of our professional task is incomplete.

In a lecture by Bill Curtis of the MCC in Austin, Texas, he pointed out that in the narrow domain of software engineering, the issues involved go far beyond the cognitive problems focused upon by computer science. As systems increase in scale by successive orders of magnitude, their construction raises additional ranges of problems, as illustrated in Table II.

Thus, although the foreground is occupied by the cognitive problems of software engineering, members of our profession must be also be competent to deal with social, managerial, organisational, economic, and political issues. Solving the cognitive problems using CASE tools, formal specification methods, and so on, will not suffice. Suppose we can get the engineering of software right, including delivering on time, on budget, and what is specified. Our work should begin with the analysis and specification tasks, and we still do not adequately understand the issues of making specifications of what the users actually need in social, managerial, organisational, economic, and political terms.

The IT systems (including but broader than software systems) function only in an organisational context. This is pictured in Fig. 5, where IT belongs within a layer of formal or bureaucratic organisation which, in turn, functions only as a component within the embracing informal human system. IT systems operate only according to formally given instructions which can be implemented in software or hardware. The formal system that lies outside the IT system may be documentation in mathematical, logical, or graphical notations or in carefully drafted prose. The formal system functions only when people use it according to very precise rules, which may be written down or simply learned and maintained by practice. Good engineering discipline creates much of the relevant formal system but we also make formal systems that we call bureaucracies. Bureaucracies can be either productive or wasteful, depending upon their design. We cannot avoid using a bureaucracy as an enabling structure within which the automated system can function, but we should design it with care because it

Table II. Problems Raised by System Scale (After Curtis, 1990)

\begin{tabular}{lll}
\hline Lines of code & Resource & Type of problem \\
\hline $1 \mathrm{k}$ & Individual & Cognitive \\
$10 \mathrm{k}$ & Team & Social \\
$100 \mathrm{k}$ & Several teams & Managerial \\
$1 \mathrm{~m}$ & Company & Organisational \\
$10 \mathrm{~m}$ & Consortium & Economic \\
$100 \mathrm{~m}$ & Nation & Political \\
\hline
\end{tabular}




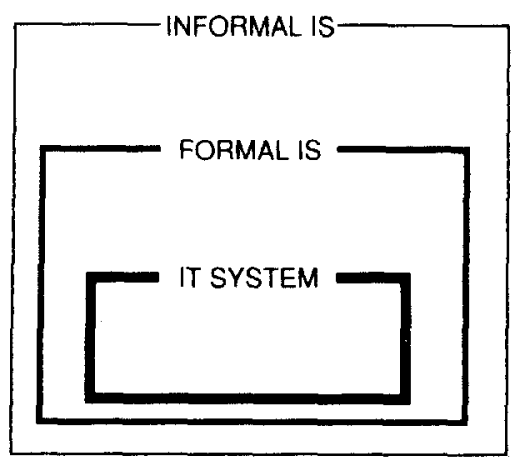

Fig. 5. The organisation as an information system. Source: Stamper (1989).

can be crucial to the effectiveness of the system as a whole. The choice of bureaucracy is an interface between the informal and technical systems, a function of the available IT and the ambient culture.

The bureaucratic layer of the organisation is the necessary intermediary between the IT system and the informal human system where data become information. Data, the mechanically made marks and signals, acquire their meanings only by virtue of the people in the informal system. Meaningful signs used with intention create relationships between people and organisational units, only at this level we are dealing with information, rather than data. One of the functions of the bureaucratic layer is to allow people to work in a rather mechanical way, performing very limited functions and operating with forms according to rules, without their having responsibility for the substantive issues. Modern IT makes it possible to change the bureaucratic layer so that people can be employed for their understanding and their ability to deal with many complex functions. Greater effectiveness can be obtained by making better use of human abilities, as a by-product of the efficiency of IT.

The informal system, as its name indicates, cannot be formalised without its ceasing to be informal. We can use formal, mathematical, or logical models to theorise about it but we have to be very careful not to forget that any formalisation can be only a parody of the richness of the informal system. The requirements of an organisation for a new information system are always rooted in the informal system and expressing those requirements consists of making them explicit in precise, formal terms that are (where appropriate) adequate to define a software engineering problem. We appear to lack a conceptual framework that will enable us to think of the whole organisation as the information system so that professionally we give as much attention to the informal system as to the computer. 


\section{A SEMIOTIC FRAMEWORK}

Three of the challenges facing our profession are to clarify the concepts we employ, thereby making it possible to create a better theoretical foundation for our work; to do this in a way that allows us to think of the organisation as the information system on which we are working; and to develop methods and tools to put these improved concepts and broader, organisational perspective into effect in our work. There will be many ways of facing these challenges, but personally, I can offer one approach. It entails placing our professional concerns within what I call the "semiotic framework."

Semiotics is the doctrine of signs. If one takes the concept of a sign as the primitive notion for building a theory of information, one can investigate the properties of signs. "Information" can then be given many different precise meanings; at least one specific meaning should be differentiated for each level of structure which we give to signs. The lowest level of these structures is that of raw physical phenomena out of which we engineer our IT hardware. At the top, we have the information structures that constitute our social world. In between we have the making of patterns that can be accurately distinguished and transmitted on a repeated basis; these empiric aspects are of great concern, for example, to communication engineers and designers of computer-human interfaces. Given reliable patterns, we can begin to organise at the syntactic level of data modelling and program writing. The physical, empiric, and syntactic levels constitute the technical platform for information systems and they are the primary focus of our profession today. If you are running an organisation, you will be more concerned with information that expresses your meaning: the semantic level. That will not be enough because anyone operating within an organisation will want to express her intentions, so the information has to be structured at an even higher, pragmatic level. Information in organisations is always intended to have a social effect if it is to have any value, and until the physical tokens (data) produce those social effects, they do not inform (create mutual understanding, agreements, commitments, shared expectations, etc.). The semiotic framework consists of these six levels of structure which unite the technical and the social aspects of our professional concerns (Fig. 6).

Information is the means by which we construct our social world, including our organisations. We begin by building a technological platform: hardware for storage, transmission, and processing; patterns that can be communicated and stored reliably; and software, records, and files to represent structures. Machinery can provide these three classes of functions. Only people can perform the next three: forming signs into meaningful expressions; attaching a "speaker's" intention to these expressions and directing them to an addressee; and interpreting the messages one receives. Thus, minute fragments of our physical reality can generate the shared understanding, values, and commitments that constitute our social reality. 


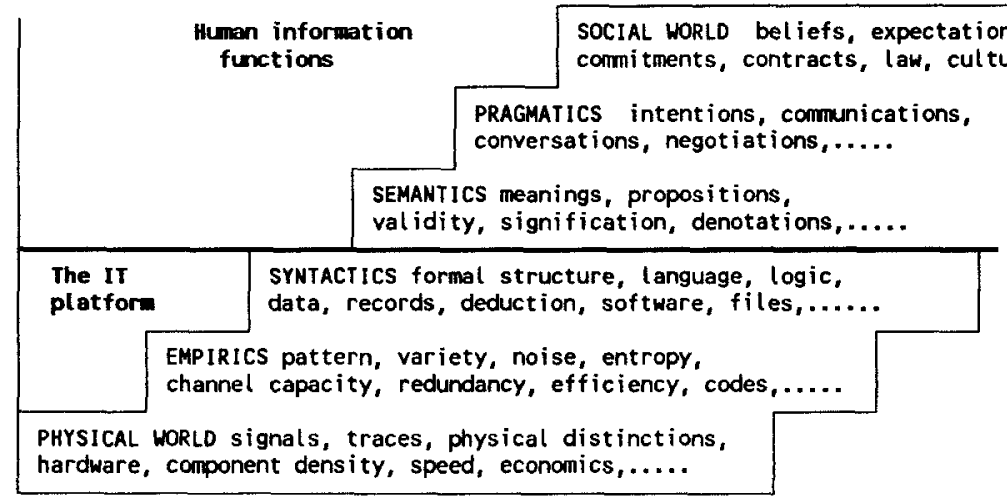

Fig. 6. The semiotic framework.

The rather crude distinction that is made between data and information may be interpreted in the semiotic framework. Signs that are well formed in their physical, empiric, and syntactic structures constitute what we loosely call "data." Data means "things that are given," so in this context, we can think of data as given signs and able to fulfil some purpose. Signs that have meaning and intention, and are capable of producing a social effect, are what we call "information.",

\section{THE NEW PROFESSIONALS}

We are now in a better position to see how our profession may change. If information and information systems are the means by which, from physical resources, we construct our social world, then the expertise in our profession should relate to all the problem areas in the semiotic framework. Some may specialise in constructing the data systems, the platform structured, reliable,

\footnotetext{
${ }^{2}$ This is a small improvement on the commonly sloppy use of these terms, but in fact, many of the commonest technical terms necessary for talking about information systems, for purposes of analysis, design or diagnostics, have different and specific meanings at each level of the semiotic framework. For example, we commonly talk of an amount of information measured in pages, bytes, or characters, which ate all measures of physical sign-tokens; the entropy measure of bits is the best-known precise measure of information and it belongs to the empirics level; Carnap and Bar-Hillel (1953) created a whole spectrum of logical measurements of information at the syntactic level (although they called them "semantic" measures); Devlin (1991) defines a unit of information called an "infon," which has some similarities to the "surrogate" in Stamper's Norma (1986) based on a semantic theory; precise, pragmatic measures of information can be constructed by observing the effect of a message on a person's likely behaviour, for example, by constructing measures based on subjective probability; finally, a social measure of information could be based on measurements of the changes made by a message to a system of social norms. For more on this topic see Stamper $(1973,1993)$.
} 
physical signalling devices on which the human organisation is then elaborated; this consists of software engineering in a broad sense. The others, perhaps we should think of them as information architects, help the users to know what information needs they have, before they create a precise definition of these requirements. The idea of making an elaborate specification of requirements, which is then handed over to a software house for implementation, will probably disappear as we learn how to help the users to evolve their own information systems (that is their organisations) on the basis of a generic IT platform. Gradually the technical aspects of our profession will be reduced from their overwhelming dominance to a balanced place in relation to the human and organisational issues where our expertise is now limited.

The new professionals must understand the relationship between IT and the social world where it serves its main functions. As architects create spaces, whilst civil engineers build structures and craftsmen fill them with walls, plumbing, etc., so must the information architects assist people to create the technical structures in which the desired social forms can be generated. Comfortable social "spaces" are informal, whilst the computer imposes formality and its accompanying penumbra of bureaucracy. The architect does not prescribe the behaviour of the occupants of a building, nor will the information architect prescribe the information functions of the IT users, but in both cases, the placing of the boundary structures has a strong influence over the human behaviour within them. We must expect that the training in information architecture will be long, just as it is in conventional architecture, because the practitioner must be competent in such a wide range of disciplines, both social and technical.

\section{THEORETICAL KNOWLEDGE TO SUPPORT INFORMATION ARCHITECTURE}

Earlier, it was suggested that the lack of suitable theories is an obstacle to developing our profession. There are no lacunae on the technical levels of the semiotic framework: electronics, telecommunications theory, and the mathematics and logic of software, etc., are highly developed and very well suited to practical work in their fields. But our theories are weak in the human and social areas of semiotics.

There are bodies of knowledge about social psychology and theories of organisation. But these are rather informal and incapable of providing the necessary formal bridge between the rigorous demands of technology and the informality of human information needs. They are helpful but not sufficient for developing computer-based systems because they cannot handle the rich and detailed formal structures which must be specified before software can be written. On the other hand, we have methodologies and specification tools that have 
evolved from the computing paradigm and are incapable of expressing concepts in the human and social domains. For example, data-flow diagrams of all kinds and schemata for navigating through complex data stores are the most important of these. Their defect is that they impose a computer metaphor on organisations, which obscures the essentially different nature of social systems. We need, I believe, an approach that can handle rich formality without distorting the picture of organisational behaviour.

There do exist some relevant theories and our profession will gradually begin to absorb them. In semantics Montague's theory (Montague, 1974; Dowty et al., 1981) is a highly developed product of mathematical linguistics but it is quite impractical and probably not at all appropriate for our purposes. Pragmatics can build on speech act theory (Austin, 1962; Searle, 1969; Searle and Vanderveken, 1985) and the logics of deontics and action [the founder (von Wright, 1963; and, e.g., Hilpinen, 1981)], which are beginning to be applied to programming (Meyer and Wieringa, 1993) and to systems analysis (Lehtinen and Lyytinen, 1986). At the social level some relevant theories are beginning to be distilled from work on computers, logic, and law (Stamper, 1980, 1986, 1993), but the drive at this semiological level is upon forcing social concepts to fit the procrustean bed of predicate logic (Stamper, 1991).

The basis of many of these interesting new theories is a kind of logic that evolved to solve problems in mathematics, worse still, a mathematics which had evolved to deal with physical phenomena, most notably astronomy and ballistics or the properties of inert matter. Those logics have captured the most important abstract structures of the physical world. It is my belief that we should build kinds of logic that capture structures in our social world.

To support and illustrate my argument, I shall explain the approach my own group has been adopting, with the qualification that it is only one (perhaps an unusually radical one) of a wide range of interesting possibilities. I feel confident to present these ideas because they prove to be very effective in practice. They lead to a protologic which we are slowly refining. Essentially it starts from the view that reality is constructed by agents acting socially. This leads to a strict semantic theory relating signs (including numbers, words, expressions, and larger linguistic units) to the behaviour of persons and groups. The behavioral options are called "affordances" (after Gibson, 1968, 1979; see also Michaels and Carello, 1981) because they are what the abilities of the agentin-its-environment will afford, but in a social context, these are established by social norms. The protologic is called "Norma." From an information systems perspective, Norma allows us to describe social formations, including organisations, as structures of social norms. The method of analysis we have developed for specifying the norms for a system is called MEASUR (Stamper, 1991b). A few of the basic concepts are presented. 


\section{THE ORGANISATION AS AN INFORMATION SYSTEM}

We treat an organisation as an information system. This is very different from considering an organisation as a signalling system. This signalling view is adopted by all the common computer-oriented methods and exemplified by the data-flow model of an organisation where different devices pass messages among themselves, store them as records, retrieve them from the store, and transform them in various ways before onward transmission to other users. A related model is the data-navigation model; here the nodes are the data elements in a storage structure and the task is to find ways of navigating through the storage space to perform various computational and data-processing tasks. Our view treats the organisation as a structure, a social form where information is a process of making, maintaining, and changing the organisational form.

The form of an organisation or any organised behaviour consists of the regularities of behaviour displayed by the members of the group. The regularities we call norms. Norms may be unconsciously held by the members as part of their shared culture. Other norms may have been agreed explicitly in conversation, some others would be written down in the form of rules for the organisation to use internally, and yet other norms will be legal ones providing a framework within which the organisation takes on its external shape in relation to other individuals and institutions in the broader society.

When we can describe the substantive tasks of an organisation in terms of the norms governing behaviour of the members, we have the kernel of the definition of an information system. The informal norms present a strange problem: if we want to express them, we cannot do so directly because the essence of an informal norm is that it is not expressed explicitly. But there is an escape. All we need to do to have access in practice to a "device" that stores and interprets informal norms is to identify the agent responsible for applying the norm. Thus the norm which governs when a person is sick and entitled to sickness benefit will be made the responsibility of a qualified medical practitioner; at the other extreme we shall write down norms that are detailed and explicit, so that, given the relevant data, their operation can be decided by an algorithm. However, the contents of these explicit norms will be expressed in a language which ultimately will have to be interpreted by responsible agents according to the informal norms of the surrounding culture.

Thus, translated into concrete terms of how we must proceed, we need a language in which we can express social norms in a way which can be understood easily by the user and simultaneously intepreted by a computer system. This language must also enable us to identify responsible agents clearly in those circumstances where algorithmic norms are not available.

Note that whereas familiar, the data-flow, data-navigation, and data-pro- 
cessing methodologies are intrinsically technical in their orientation; this new norm-oriented approach makes no tacit assumptions of a technical kind-it is purely confined to representing organisational and social issues.

\section{AN ILLUSTRATION}

This small example (known as the CRIS Case) concerns a learned society which runs conferences from time to time. The society needs an organisation to support this activity. The most limited solution possible is merely to state that we have a Society, and one of its forms of behaviour (affordances) is to hold conferences. This we would represent by

Society conference

which indicates that a responsible group, the Society, can behave in such a way as to produce instances of the type conference. How the Society behaves in order to make these conferences happen is entirely a matter for its own informal system.

This is a very weak description, you may say. Although that is true in one sense, no description, however elaborate, can avoid our having to admit that only a small part of the real organisation can be represented, and the rest has to be handed over to the discretion of someone or some group in the informal system.

Let us try to strengthen the description. The first step entails defining the structure of responsible teams and individuals, together with the relevant actions they can perform, as in Fig. 7. This constitutes a semantic schema where the arcs such as $x \ldots y$ mean that an instance of $y$ can exist only when an appropriate instance of $x$ exists. Thus the schema includes the logically necessary time constraints. It is no trivial matter to construct such a schema: a little exercise is left for the reader because there is no antecedent for work-try some alternatives such as conference, learned-society, person, and society, by asking whether or not, for practical purposes, a work depends for its existence upon any one of these. (Incidentally, this schema can be implemented directly in the Normbase we have developed to create a computer system to support the business system specified.)

The next step is to specify the agents responsible for each element in this structure. For example, the person who contributes a report that reviews a paper will be responsible for it and the programme committee will be responsible for a paper being selected. These are probably decisions that we shall always wish to keep within the ambit of human discretion and never bureaucratise.

The information in Fig. 7 depicts the visible framework of the informal system. Beneath it we can find the norms we cannot, or choose not to, make 


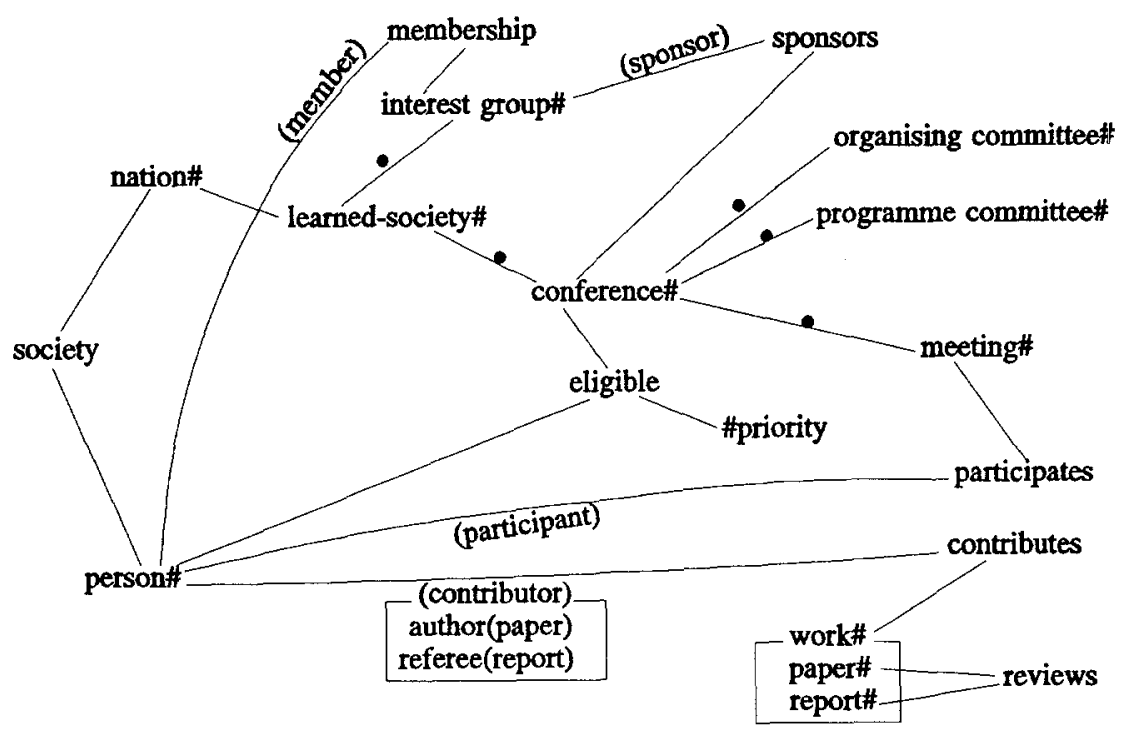

Fig. 7. Semantic schema.

explicit by locating the agents who know those norms and put them into operation. Upon the framework we can construct a system of explicit norms or rules.

The authority for an organisational act may not be directly assigned to a single agent. Often a rule is used to divide responsibility among agents with different roles to play. At this stage we would write rules and regulations (or even legislation). We are working on two languages for specifying these norms. NORMA is the basic language, in which the norm structure is unambiguously represented, but to manipulate it we use LEGOL, which is intended to be fairly easy to understand and very compact because it can be ambiguous. Here is a simple example:

\section{(member (organising committee orwhile program committee) orwhile contributor (selected (paper))) then priority 1 (member orwhile contributor)}

which says that any member of the organising or programming committee or any author of a selected work has priority 1, the symbol "orwhile" being a kind of logical "or" that takes account of time. The then operator bears no resemblance to the "if. . .the" of propositional logic because its validity is not a function of the validity of its parts; it creates a norm with an existence independent of its components.

Here is another example, which depends upon the first and involves writing two simple norms that define "author" and "referee." It also employs the 
symbols "while and "whilenot," which are somewhat like "and" and "not" with time aspects. It makes use of a context limiter "for Conference" (which, in effect, interpolates "while conference" to select only relevant data for one case) that limits the rule to a single conference:

contributor (paper) then author

contributor (report) then referee

for conference

(member (interest-group while sponsor)

orwhile author orwhile referee) whilenot priority 1 (person)

then priority 2 (person)

We can define who is eligible as any person belonging to the sponsoring interestgroup plus those who have signalled their knowledge of the field by contributing abstracts: for interest-group while conference

(member (interest-group of sponsor) orwhile contributor (abstract)) then eligible (person)

We can continue this analysis until we have defined enough rules for our purpose and assigned to suitable groups or persons responsibility for the other norms. We can also go on to deal with what happens when the rules are not obeyed, which entails writing more rules or making someone responsible for applying the necessary sanctions.

The rules themselves we assume are understood (in their natural language form) by the members of the Society, its committees, and their officers, who apply them through the informal system. At this point we have no bureaucracy.

When it comes to designing the bureaucratic or technical system, we have the necessary information here, in this limited description of the informal system. Once we have assigned responsibility for applying them, the rules themselves tell us who has to pass information to whom.

As a point of comparison with the existing methods, notice that we have said nothing about data flows but we have all the necessary information from which they can be deduced if we know the rules and the responsible agents. This method not only is truer to the organisational reality, but is far more compact than the familiar methods (Liu et al. report massive reductions in documentation and increased comprehension by users). Notice that this approach also includes the means of analysing meanings and intentions, so it does provide a way of approaching the difficult theoretical areas in which our information architects will have to work.

Lest there be any misunderstanding, these methods do not imply that the information systems architect would be engaged in constructing some Orwellian nightmare. On the contrary, they respect the individuals and groups who are 
responsible for the organised activity. The analysis can be used just as easily for dismantling, as for constructing formal bureaucratic structures: the norms are handed over to responsible agents. This is unlike the methods current today, which tend to encourage the growth of electronic Taylorism, formalising, and automating are their purpose. The data-flow, data-navigation, and data-processing approaches do not include explicit techniques for reducing bureaucracy and automation. Too often, our profession has played the role of surrogate IT salesmen simply because our methods have a built-in tendency to suggest automation. Our job is as much to enrich our informal information systems as to enable us to use IT where appropriate.

It is possible to discuss organisations as information systems in a manner that is detached even from any reference to data flow or data manipulation, that is, quite apart from any shadow of IT. This fact gives me confidence that eventually we shall evolve as rich a theoretical basis for the architecture of information systems as we now have for the study of computer languages and the formal aspects of software engineering. Many methods besides those illustrated here will equip this new branch of our profession.

\section{INSTITUTIONAL INERTIA}

So we can develop adequate concepts and methods for working on the semantic, pragmatic, and social aspects of our problem domain. But that is no guarantee that change will take place.

There remains another major obstacle, which is the present institutional framework. This does not help and sometimes tends to impede the adoption of the relevant ideas. The key institutions are manifold: the IT industry, the consumers of IT, the managers of IT, our own profession, the government, the university system, the schools, and the law are perhaps the major ones. Let us examine them briefly as a way of summing up and proposing a course of action.

The IT industry itself, being concerned with its efficiency-promoting products, leaves the consuming organisations to take care of the effectiveness questions themselves. I do not think that they are to blame for adopting this stance; any other would be too costly. They could help to promote change in two ways. One would be to set about marketing solutions more deliberately. This would entail growing or acquiring strong consultancy wings, which would have to operate with a degree of independence from the sale of hardware and software to avoid their solutions being dismissed as merely a sales instrument for IT products. This may be too difficult a balancing act to perform. The second would be to lobby more strongly in favour of other institutional changes, especially in national government policies, European Union initiatives, structures, and curricula in educational institutions.

The consumers of IT are scarcely organised as such except as lobbies to 
match the various computer companies. They need to have a stake in some major research programmes to investigate why IT has brought them surprisingly small benefits. They, too, should lobby others to support this action, and their best form of lobbying would be to spend some of their own funds to encourage more information systems research and development which focuses on organisational issues. They are the principal beneficiaries.

Consumers of IT can also give responsibility in the matters of IT investment to professionals who are concerned with the organisation and treat the technology as a means to an end. Today their appointees are still predominantly technically orientated and tend to see themselves as managers of IT facilities. Ideally there should be a pool of skilled people with the necessary balance of knowledge and experience, but these are still rather rare. Once again, this is a score on which companies should start to lobby the universities. Our profession can give a stronger lead than it has in the past.

The government, as always in the birth of new institutions, has the greatest power. In many ways it can be midwife at the rebirth of our profession. For example, although, for some years now, we have been operating in the information economy, where the production and distribution of knowledge and information account for most of our wealth production, we still structure our national economic statistics as we did to suit a world dominated by physical production and distribution. A change in the way economic facts are presented would alone direct the attention of policy-makers to issues that currently remain hidden in the figures.

Education can be reshaped by government but the universities are the institutions that are best able to exert a strong influence for change. They are, in the main, structured and funded by government in ways that tend to preserve the status quo. But the universities have enough autonomy to break out of those limitations. Perhaps the greatest distortion is the funding of those subjects that could best help to create the new branch of the profession: those concerned with the use of computers and communications in business and administration. The teachers and students in the subject areas needed to enrich our profession are forced to work without the necessary rich IT environment. In most countries they are funded along with the humanities as people who are permitted to write and talk about computers but not use them.

Schools have less room for manoeuvre but the makers of curriculum experiments could be giving more thought to the knowledge needed in society before we can all make effective use of the computer as anything but a computer. (Let us change this misleading name to something better, such as a "communicator"') The humanities might have far more importance in economic terms than they are credited with today if they were taught as basic skills for the use of the IT. I have found that a small step can be made by teaching a curriculum based on the semiotic framework introduced above. This at least allows one to 
think analytically and critically about the multimedia technologies that will increasingly bombard us daily. I believe that the semiotic concepts are basic for the new branch of our profession.

\section{CONCLUSION}

IT is not bringing the economic benefits we might reasonably expect. The cause seems to be an unbalanced preoccupation with the technological aspects of IT compared with the organisational aspects that unleash its value in society. We need a new balance in our profession.

\section{REFERENCES}

Austin, J. L. (1962). How to Do Things with Words, Oxford University Press, Oxford. Carnap, R., and Bar-Hillel, Y. (1953). Semantic information. Br. J. Phil. Sci. 4, 147-157.

Cron, W. L., and Sobol, M. G. (1983). The relationship between computerization and performance: A strategy for maximizing the economic benefits of computerization. Inform. Manage. 6.

Devlin, K, (1991). Logic and Information, Cambridge University Press, Cambridge.

Dowty, D. R., Wall, R. E., and Peters, S. (1981). Introduction to Montague Semantics, Reidel, Dordrecht.

Franke, R. H. (1987). Technological revolution and productivity decline: Computer introduction in the financial industry. Technol. Forecast. Social Change 31, 143-154.

Gibson, J. J. (1968). The Senses Considered as Perceptual Systems, Allea \& Urwin, London.

Gibson, J. J. (1979). The Ecological Approach to Visual Perception, Houghton Mifflin, Boston.

Hilpinen, R. (1986). New Studies in Deontic Logic, D. Reidel, Dordrecht.

Jayaratna, N. (1990) Systems analysis: The need for a better understanding. Int. J. Inform. Manage. 10, 228-234.

Lehtinen, E., and Lyytinen, K. (1986). An action based model of information systems. Inform. Syst. 13(4), 299-318.

Meyer, J.-J. Ch., and Wieringa, R. J. (eds.) (1993). Deontic Logic in Computer Science, Wiley, Chichester and New York.

Michaels, C. F., and Carello, C. (1981). Direct Perception, Prentice-Hall, Englewood Cliffs, NJ. Montague, R. (1974). In Thomason, R. H. (ed.), Formal Philosophy, Yale University Press, New Haven, CT.

Niblett, B. (1980). Computer Science and Law, Cambridge Universiry Press, Cambridge.

Scott-Morton, M. S. (1989), Management in the 1990s: The changing role of information technology. In Niet bij Informatica alleen, Jaarcongres '89, NGI, Amsterdam.

Searle, J. R. (1969). Speech Acts-An Essay in the Philosophy of Language, Cambridge University Press, Cambridge.

Searle, J. R., and Vanderveken, D. (1985). Foundations of lllocutionary Logic, Cambridge University Press, Cambridge.

Stamper, R. K. (1973). Information, Wiley \& Sons, New York, and 2nd ed., Blackwell, Oxford (forthcoming).

Stamper, R. K. (1980). LEGOL: Modelling legal rules by computer. In Niblett, B. (ed.), Computer Science and Law, Cambridge University Press, Cambridge.

Stamper, R. K. (1986). A logic of social norms for the semantics of business information. In Steel, T., and Meersmann, R. (eds.), Knowledge and Data, North-Holland, Amsterdam. 
Stamper, R. K. (1991), The role of semantics in legal expert systems and legal reasoning. Ratio Juris 4(2), 219-244.

Stamper, R. K. (1991b), Social norms in requirements analysis-an outline of MEASUR. In Jirotka, M. and Goguen, J. (eds.), Requirements Engineering: Technical and Social Aspects, Academic Press, London and New York.

Stamper, R. K. (1996). Signs, Information, Norms and Systems. In Holmqvist, B. and Andersen, P. B. (eds.), deGruyter, Berlin.

Stamper, R. K., and Liu, K. (1993). Generating Information Systems as a By-Product of the Legislative Process, Proceedings, Conference on Computers and Law, Florence, Dec.

Steel, T., and Meersmann, R. (1986). Knowledge and Data, North-Holland, Amsterdam.

Strassmann, P. A. (1985). Information Payoff, Free Press, New York.

Strassmann, P. A. (1990). The Business Value of Information Technology, Strassmann, New Canaan, CT.

von Wright, G. H. (1963). Norm and Action, Routledge and Kegan Paul, London. 